Design and Subjects We studied injury death statistic of about $90 \%$ of total 10000 communes in 2005-2008 and conducted indept-interview with 42 health staff and 70 family members of death cases due to injuries.

Results Injury mortality decreased from 45.01/100 000 (year-1) in 2005 to $4384 / 100000$ (year-1) in 2008. The 20-59 group had the highest proportion of injury mortality, followed by group of over 60. Road traffic injuries were the leading cause of death among those 15 aged and over. Drowning accounted for the highest injury mortality among those under 15 . Poisoning was more common in the 20 and more age group. Falling accounted for the highest proportion of injury mortality in the 60 and more age group. Proportion of village health workers trained on recording injury mortality was low. Causes of death had not been identified and classified correctly in many communes.

Conclusions and Recommendations This study provides use baseline information on injury mortality. The results reveal that death due to injury caused mainly by road traffic injuries and drowning and the quality of injury mortality data recording was poor and needs to be improved.

\title{
0336 INJURY MORTALITY SURVEILLANCE IN VIETNAM: RESULTS AND RECOMMENDATIONS
}

T L H Nguyen*, L T T Ngoc Correspondence: General Department of Preventive Medicine and Environment, Occupational health-Injury prevention Division, $\mathrm{MOH}$, Alley 135 Nui Truc, Ba Dinh district, Hanoi city, Vietnam

10.1136/ip.2010.029215.336

Background In Vietnam, the rate of deaths due to injury was three times as high as that of deaths due to infectious diseases and injury is the leading cause of death in children under 18. Injury surveillance system was set up in 2005.

Objectives To assess injury mortality and quality of injury mortality data recording. 